Purdue University Purdue e-Pubs

$9-1-2005$

\title{
High-performance carbon nanotube field-effect transistor with tunable Polarities
}

\author{
Yu-Ming Lin \\ Joerg Appenzeller \\ Birck Nanotechnology Center, Purdue University, appenzeller@purdue.edu \\ Joachim Knoch \\ Phaedon Avouris
}

Follow this and additional works at: http://docs.lib.purdue.edu/nanodocs

Lin, Yu-Ming; Appenzeller, Joerg; Knoch, Joachim; and Avouris, Phaedon, "High-performance carbon nanotube field-effect transistor with tunable Polarities" (2005). Other Nanotechnology Publications. Paper 8.

http://docs.lib.purdue.edu/nanodocs/8

This document has been made available through Purdue e-Pubs, a service of the Purdue University Libraries. Please contact epubs@purdue.edu for additional information. 


\title{
High-Performance Carbon Nanotube Field-Effect Transistor With Tunable Polarities
}

\author{
Yu-Ming Lin, Member, IEEE, Joerg Appenzeller, Senior Member, IEEE, Joachim Knoch, and \\ Phaedon Avouris, Member, IEEE
}

\begin{abstract}
State-of-the-art carbon nanotube field-effect transistors (CNFETs) behave as Schottky-barrier-modulated transistors. It is known that vertical scaling of the gate oxide significantly improves the performance of these devices. However, decreasing the oxide thickness also results in pronounced ambipolar transistor characteristics and increased drain leakage currents. Using a novel device concept, we have fabricated high-performance enhancement-mode CNFETs exhibiting n- or p-type unipolar behavior, tunable by electrostatic and/or chemical doping, with excellent OFF-state performance and a steep subthreshold swing $(S=63 \mathrm{mV} / \mathrm{dec})$. The device design allows for aggressive oxide thickness and gate-length scaling while maintaining the desired device characteristics.
\end{abstract}

Index Terms-Carbon nanotube, doping, field-effect transistor, Schottky barrier (SB).

\section{INTRODUCTION}

$\mathbf{S}$ INCE THE discovery of carbon nanotubes in the early 1990s [1], [2], the field has witnessed an immense growth. Due to the small diameter $(\sim 1 \mathrm{~nm})$, nanotubes are ideal candidates to study one-dimensional (1-D) electrical transport phenomena, even at room temperature. In particular, single-walled carbon nanotubes, which consist of a single layer of a graphene sheet wrapped up to form a seamless tube [3], possess exceptional electrical properties, such as high current carrying capability [4] and excellent carrier mobility [5]. Both theory and experiments have demonstrated that these tubes can be either metallic or semiconducting, depending on the chirality of their atomic structure with respect to the tube axis. Using semiconducting nanotubes, carbon nanotube field-effect transistors (CNFETs) have been first realized and reported in [6] and [7]. These early CNFETs, however, showed poor device characteristics. Since then, remarkable progress has been made to improve the performance of CNFETs by approaches such as: 1) reducing the gate-oxide thickness [8]; 2) adopting high- $\kappa$ dielectrics for the gate oxide [9]-[11]; 3) using an electrolyte as the gate [12], [13]; and 4) reducing contact resistance by choosing proper contact metals [14] or post-process treatments [15]. These techniques have significantly advanced CNFET devices to exhibit characteristics rivaling those of state-of-the-art Si-based MOSFETs. With the scaling limit of conventional

Manuscript received August 12, 2004; revised December 17, 2004.

Y.-M. Lin, J. Appenzeller, and P. Avouris are with the IBM T. J. Watson Research Center, Yorktown Heights, NY 10598 USA (e-mail: yming@us.ibm.com; joerga@us.ibm.com; avouris@us.ibm.com).

J. Knoch is with the Institute of Thin Film and Interfaces and Center of Nanoelectronic Systems, Research Center Jülich, D-52454 Jülich, Germany.

Digital Object Identifier 10.1109/TNANO.2005.851427 (a)

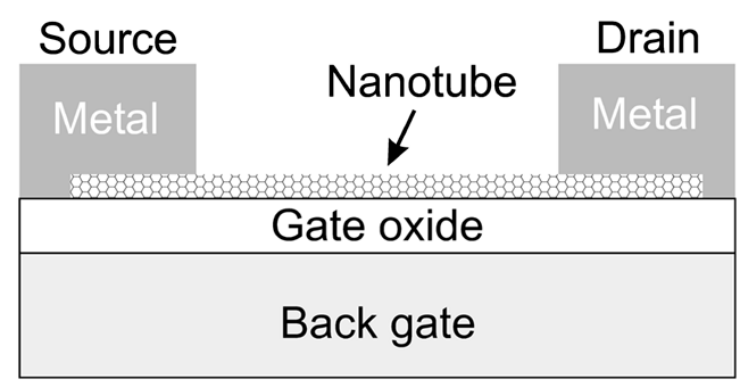

(b)

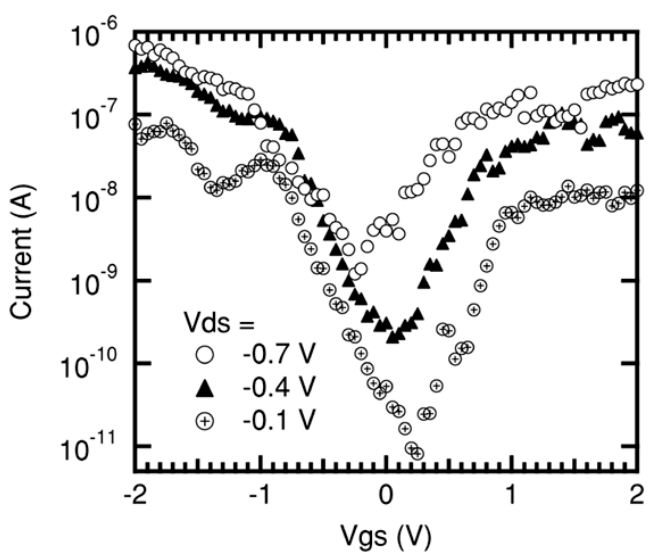

Fig. 1. (a) Schematics of a CNFET with a back-gate configuration (b) Measured subthreshold characteristics $I_{d}\left(V_{\mathrm{gs}}\right)$ of a typical CNFET (nanotube diameter $\sim 1.4 \mathrm{~nm}$ ) at different drain voltages. The gate oxide consists of a layer of thermally grown $\mathrm{SiO}_{2}$ (10-nm thick), and the contacts are made of Ti. The channel length between the source and drain contacts is approximately $300 \mathrm{~nm}$.

CMOS in sight [16], carbon nanotubes show great promise among the materials investigated so far for post-Si technology.

Most CNFETs studied so far have adopted a back-gate top-contact geometry (see [17] and [18] for a review), as shown in Fig. 1(a). In this back-gate configuration, the nanotubes are dispersed or grown on a conducting substrate covered by an insulating layer. Two metal contacts are deposited on the nanotube to serve as source and drain electrodes, while the conducting substrate is the gate electrode in this three-terminal device. This CNFET structure, albeit simple, has been extensively studied to provide invaluable insights and understanding of fundamental properties of nanotubes. However, these $\mathrm{CN}$ FETs are found to possess several unfavorable features that severely constrain their usefulness in vital applications such as CMOS-like circuits. In order to address the disadvantages of a simple back-gated CNFET, in this paper, we present a novel 
(a)

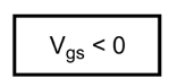

(b)
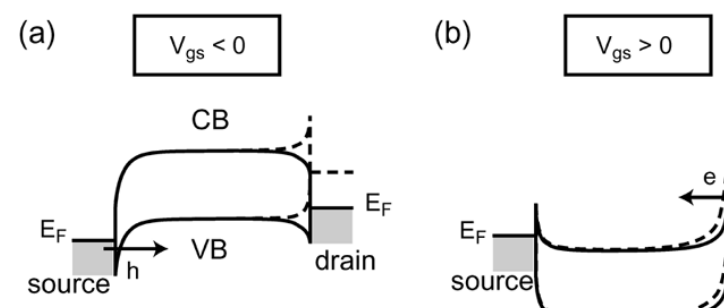

$\mathrm{CB}$ : conduction band

VB: valence band$$
\mathrm{V}_{\mathrm{ds} 2}
$$

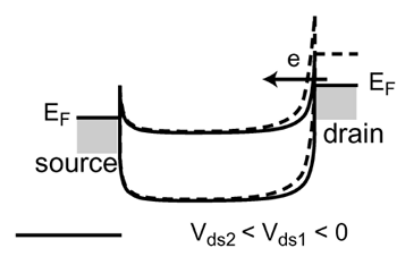

Fig. 2. Schematic band diagrams of a CNFET for: (a) $V_{\mathrm{gs}}<0$ and (b) $V_{\mathrm{gs}}>0$. Two different drain voltages $V_{\mathrm{ds} 1}$ and $V_{\mathrm{ds} 2}\left(V_{\mathrm{ds} 2}<V_{\mathrm{ds} 1}<0\right)$ are represented by solid and dash lines, respectively.

device concept for CNFETs. In particular, our device design is capable of producing pure $\mathrm{n}$ - and/or p-type CNFETs with improved OFF-state performance and abrupt switching behavior close to theoretical limits.

First we review important electrical characteristics of the back-gated CNFET, as shown in Fig. 1(a). The understanding of operation principles and physics of this device is indispensable in order to obtain desired functionality and further improvements. Fig. 1(b) shows the subthreshold characteristics, the drain current $I_{d}$ measured as a function of gate voltage $V_{\mathrm{gs}}$, of a back-gated CNFET for different drain voltages $\left(V_{\mathrm{ds}}\right)$. For all the measurements presented in this paper, the source electrode is grounded. This CNFET device is fabricated on a heavily p-doped Si substrate covered by a thin layer (10-nm thick) of thermally grown $\mathrm{SiO}_{2}$ with titanium (Ti) contacts. In Fig. 1(b), two distinct features are observed. First, as the gate voltage increases from negative values, the current decreases, reaching a minimum value of $I_{\mathrm{off}}=8 \times 10^{-12}, 2 \times 10^{-10}$, and $10^{-9} \mathrm{~A}$ for $V_{\mathrm{ds}}=-0.1,-0.4$, and $-0.7 \mathrm{~V}$, respectively, and then rises again, exhibiting an ambipolar transistor characteristic. Second, as the drain bias becomes more negative, the minimum current $I_{\text {off }}$ increases exponentially and the gate voltage corresponding to this minimum current shifts to more negative values, showing a strong $V_{\mathrm{ds}}$-dependent $I_{\text {off }}$.

In order to understand the results of Fig. 1(b), Fig. 2(a) and (b) depicts schematic band diagrams of a CNFET for negative and positive $V_{\mathrm{gs}}$, respectively. We note that, different from a conventional MOSFET, switching of a CNFET is dominated by the modulation of Schottky barriers (SBs) formed at the nanotube/metal contacts [10], [15], [19], [20]. As indicated in Fig. 2(a) and (b), at sufficiently negative and positive gate voltages, SBs are effectively thinned to enable hole injection $\left(V_{\mathrm{gs}}<0\right)$ from the source and electron injection $\left(V_{\mathrm{gs}}>0\right)$ from the drain contact into the nanotube, respectively. Compared to the tunneling phenomena in a three-dimensional (3-D) bulk device, this tunneling current in CNFETs can be quite significant because a barrier width $\left(\sim\right.$ depletion length $\left.L_{D}\right)$ as small as a couple of nanometers can be obtained for a ultrathin body object, such as a nanotube, when thin gate dielectrics are used [21]. We also notice that, in Fig. 2, there is little band bending in the nanotube body because the carrier mean-free path in nanotubes can be as long as $\sim 500 \mathrm{~nm}$, even at room temperature [22], [23], so that carrier transport is nearly ballistic for the nanotube channel length $(\sim 300 \mathrm{~nm})$ considered here. In Fig. 1(b), the left- and right-hand-side current branches with respect to the minimum current $I_{\text {off }}$ are, therefore, contributions by hole and electron currents, and are referred to in the following as p- and n-branches, respectively. At the minimum current $I_{\text {off }}$, the hole current is equal to the electron current. Since the electron current is due to the tunneling at the drain contact, the n-branch exhibits a much stronger $V_{\mathrm{ds}}$ dependence than the p-branch (see Fig. 2 for two different $V_{\mathrm{ds}}$ 's), resulting in a $V_{\mathrm{gs}}$ shift of the n-branch as a function of $V_{\mathrm{ds}}$ and a strongly $V_{\mathrm{ds}}$-dependent $I_{\text {off }}$, as shown in Fig. 1(b). All these electrical characteristics of CNFETs have been studied previously, and detailed discussions can be found in [20], [24], and [25].

Both simulations and experimental results have shown that the switching performance of CNFETs, measured by the inverse subthreshold slope $S=\left(d \log I_{d} / d V_{\mathrm{gs}}\right)^{-1}$, and the ON-state current can be improved by decreasing the gate-oxide thickness [8]-[11], [26]. However, the enhanced gate control in a CNFET always also results in a higher $I_{\text {off }}$ with a more pronounced $V_{\mathrm{ds}}$ dependence, as explained earlier [24]. In addition, from the application's point-of-view, both n- and p-type transistors with unipolar characteristics are required for CMOS-like logic circuits, while thin-oxide CNFETs are usually ambipolar, as shown in Fig. 1(b). In order to advance nanotubes for future transistor technology, these disadvantages associated with vertical scaling have to be overcome. We have recently demonstrated the conversion of an ambipolar CNFET to a unipolar transistor using a partially gated structure where a trench is created near one of the contacts to eliminate drain leakage [27]. However, it was also found that this partially gated structure results in a reduced ON current and is not suitable for scaling to small lateral lengths due to fringing field impacts.

In this paper, we present a novel device concept for $\mathrm{CN}$ FETs using dual gates to eliminate ambipolar characteristics and create pure $n$ - and/or p-type devices with excellent OFF-state performance and a steep subthreshold swing. This is achieved by a combination of electrostatic and/or chemical doping along the tube channel. By introducing a distinct $\mathrm{p} / \mathrm{i} / \mathrm{p}$ or $\mathrm{n} / \mathrm{i} / \mathrm{n}$ band structure along the nanotube (where n, p, and i represent an n-doped, a p-doped, and an undoped (intrinsic) nanotube segment, respectively), we have successfully modified the switching mechanism of our CNFET [28]. Instead of contact switching by modulating SBs, our device operates by varying the band potential in the middle portion of the nanotube body, called bulk switching, and achieves an inverse subthreshold slope $S \sim 63 \mathrm{mV} / \mathrm{dec}$, the smallest value reported for CNFETs to date. Furthermore, this unique device structure affords aggressive gate-length scaling without deteriorated device performance.

\section{Novel DuAL-GATE CNFET DesigN}

Fig. 3 shows the scanning electron microscopy (SEM) image, as well as the device cross section of a CNFET with the proposed dual-gate structure [28]. Different from the back-gated CNFET shown in Fig. 1(a), the dual-gate CNFET possesses an additional $\mathrm{Al}$ gate electrode placed underneath the nanotube between the source and drain contacts. To fabricate the dual-gate CNFET, the Al gate (20-nm thick and $\sim 200$-nm wide) is first deposited on a p-doped $\mathrm{Si}$ substrate covered with 10 -nm-thick $\mathrm{SiO}_{2}$, followed by the oxidation of $\mathrm{Al}$ metal in moisturized oxygen at $\sim 160^{\circ} \mathrm{C}$ 


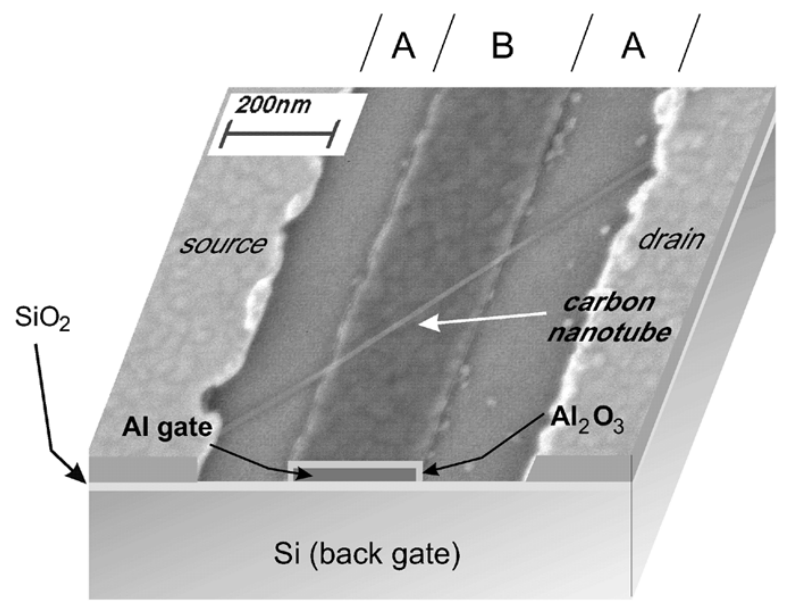

Fig. 3. Composite of the device layout of a dual-gate CNFET, showing the SEM image of a CNFET with an Al middle gate underneath the nanotube [28]. The area between the $\mathrm{Al}$ gate and the source/drain is denoted as region $\mathrm{A}$, and the $\mathrm{Al}$ gate is denoted as region $\mathrm{B}$.

for $1.5 \mathrm{~h}$ to form a thin layer of $\mathrm{Al}_{2} \mathrm{O}_{3}(\kappa \simeq 5)$. Both $C-V$ and ellipsometry measurements of the $\mathrm{Al}_{2} \mathrm{O}_{3}$ layer indicate an oxide thickness of approximately $4 \mathrm{~nm}$, in agreement with the result reported in [9]. Nanotubes produced by laser ablation [29] with an average diameter of $\sim 1.4 \mathrm{~nm}$ are then spun onto the substrate from solution. Finally, source/drain contacts made of Ti are deposited on the nanotube, each with a spacing of $\sim 200 \mathrm{~nm}$ relative to the $\mathrm{Al}$ gate. For the following discussions, the $\mathrm{Al}$ gate region is denoted as region $\mathrm{B}$, and the area between the $\mathrm{Al}$ gate and the source/drain contacts are denoted as regions A (see Fig. 3).

In our design, the $\mathrm{Al}$ gate is the primary gate that governs the electrostatics and the switching of the nanotube bulk channel in region B, while the SBs at the nanotube/metal contacts are controlled by the Si back gate (substrate), which also prevents the electrostatics in region A from being influenced by the $\mathrm{Al}$ gate. It was previously observed that carrier injection at the contacts may still be modulated by a middle gate due to fringing field impacts [11], [27]. To verify that the SBs at the contacts are not affected by the $\mathrm{Al}$ gate voltages in our design, Fig. 4 shows the measured current as a function of $\mathrm{Al}$ gate voltage $V_{\mathrm{gs}-\mathrm{Al}}$ when the $\mathrm{Si}$ back gate $V_{\mathrm{gs}-\mathrm{Si}}$ is kept floating. We find that our device is always OFF with a low current in the noise level $(\leq \mathrm{pA})$, independent of $V_{\mathrm{gs}-\mathrm{Al}}$, indicating an insignificant impact of the $\mathrm{Al}$ gate fields at the contact regions. The result in Fig. 4 implies that our CNFET can operate as a bulk-switched transistor rather than an SB CNFET since the switching using the $\mathrm{Al}$ gate does not impact the contact SBs.

CNFETs with potential profiles similar to our device have been previously fabricated by using one or more middle gates on top of the nanotube [11], [22], [32]. In those CNFETs, the nanotube is sandwiched between the top gate(s) and the back gate, and the back-gate field still contributes to the electrostatics inside the nanotube, resulting in a lower efficiency of the top gate when the back gate is kept at any constant voltage. ${ }^{1}$ In contrast, the nanotube potential of region B in our design is exclusively

${ }^{1}$ For example, the device studied in [32] has used 8-nm-thick $\mathrm{HfO}_{2}(\kappa \simeq 20)$ and 10 -nm-thick $\mathrm{SiO}_{2}(\kappa \simeq 4)$ layers as the gate insulator for top and back gates, respectively. In this geometry, the top gate efficiency is expected to be approximately $86 \%$

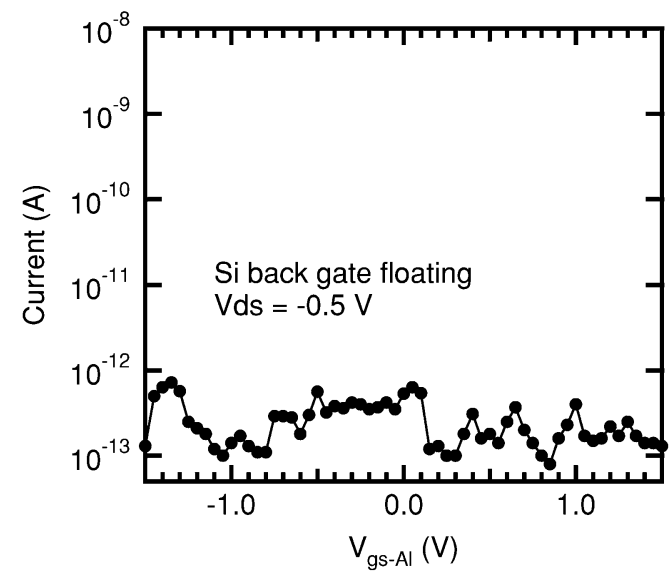

Fig. 4. Measured drain current as a function of $\mathrm{Al}$ gate voltage $V_{\mathrm{gs}-\mathrm{Al}}$ with the Si back-gate floating.

determined by the $\mathrm{Al}$ gate because the $\mathrm{Al}$ metal layer screens the field from the Si gate, giving rise to an ideal switching behavior. Another important advantage of our design is the possibility of introducing a well-defined chemical doping profile along the nanotube to further tailor transistor characteristics (see Section II-B), while no chemical doping profile can be easily introduced in a completely top-gated CNFET.

\section{A. Electrostatic Doping Effect}

Here, we present electrical characteristics of our dual-gate CNFETs at various gate voltage $\left(V_{\mathrm{gs}-\mathrm{Al}}\right.$ and $\left.V_{\mathrm{gs}-\mathrm{Si}}\right)$ configurations. In particular, we utilize electrostatic doping effects in $\mathrm{CN}$ FETs to eliminate ambipolar characteristics in an SB CNFET and to obtain a bulk-switched transistor possessing a tunable polarity ( $\mathrm{n}$ or $\mathrm{p}$ ), steep subthreshold swing, excellent OFF-state performance, and suppressed $V_{\mathrm{ds}}$ dependence. ${ }^{2}$ This is achieved by a distinct $\mathrm{n} / \mathrm{i} / \mathrm{n}$ or $\mathrm{p} / \mathrm{i} / \mathrm{p}$ band profile in our dual-gate CNFET.

Fig. 5(a) shows the subthreshold swing of a dual-gate CNFET at $V_{\mathrm{ds}}=-0.6 \mathrm{~V}$ when the two gate voltages are equal $\left(V_{\mathrm{gs}-\mathrm{Si}}=V_{\mathrm{gs}-\mathrm{Al}}\right)$. In this gate voltage configuration, the device is essentially equivalent to a standard back-gated CNFET, as shown in Fig. 1(a), and the switching behavior is mainly determined by the SBs at the contacts, resulting in the expected ambipolar behavior. In Fig. 5(a), the OFF current $I_{\text {off }}$ is below our instrument sensitivity level $\left(10^{-13} \mathrm{~A}\right) .^{3}$ The dual-gate CNFET possesses drastically different subthreshold characteristics $\left(I_{d}\right.$ measured as a function of $\left.V_{\mathrm{gs}-\mathrm{Al}}\right)$ when the Si gate voltage $V_{\mathrm{gs}-\mathrm{Si}}$ is kept constant. As shown in Fig. 5(b), the same dual-gate CNFET exhibits clear p- and n-type unipolar properties for $V_{\mathrm{gs}-\mathrm{Si}}=-2 \mathrm{~V}(\bullet)$ and $+1.6 \mathrm{~V}(\boldsymbol{\nabla})$, respectively. In Fig. 5(b), positive $(+0.6 \mathrm{~V})$ and negative $(-0.6 \mathrm{~V})$ drain voltages are used for $\mathrm{n}$ - and p-type branches, respectively, in order to illustrate regular n- and p-FET device operations.

\footnotetext{
${ }^{2}$ Electrostatic doping effects in carbon nanotubes have also been recently exploited to produce $\mathrm{p}-\mathrm{n}$ junction diodes exhibiting current rectifying characteristics [33].

${ }^{3}$ We have observed that some CNFET's possess an OFF current $I_{\text {off }}$ much smaller than others with a similar tube diameter, which cannot be explained in the context of the SB model. This behavior, however, can be understood by explicitly considering that nanotube segments are, in fact, located underneath the metal contacts and that it is these tube segments that are responsible for current injection into the gated part of the CNFET [34]
} 
(a)

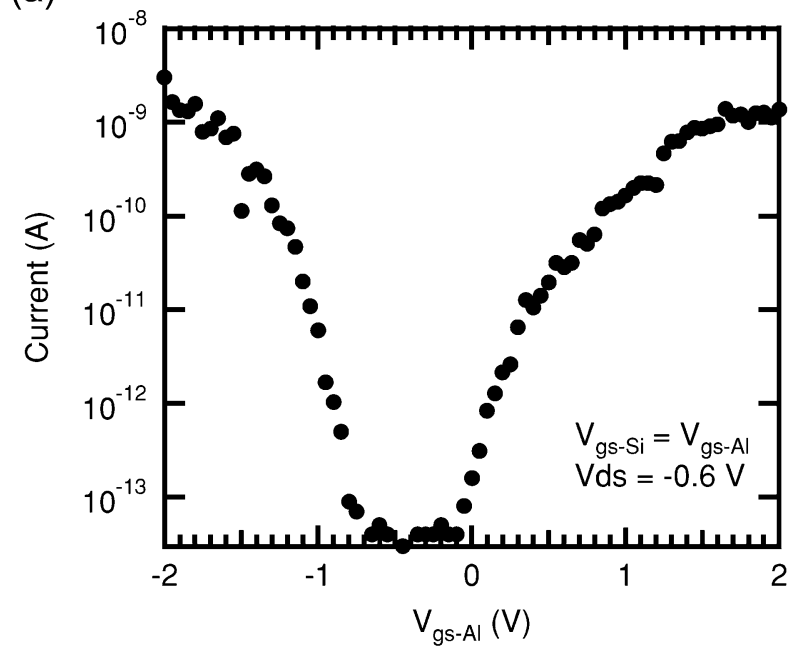

(b)

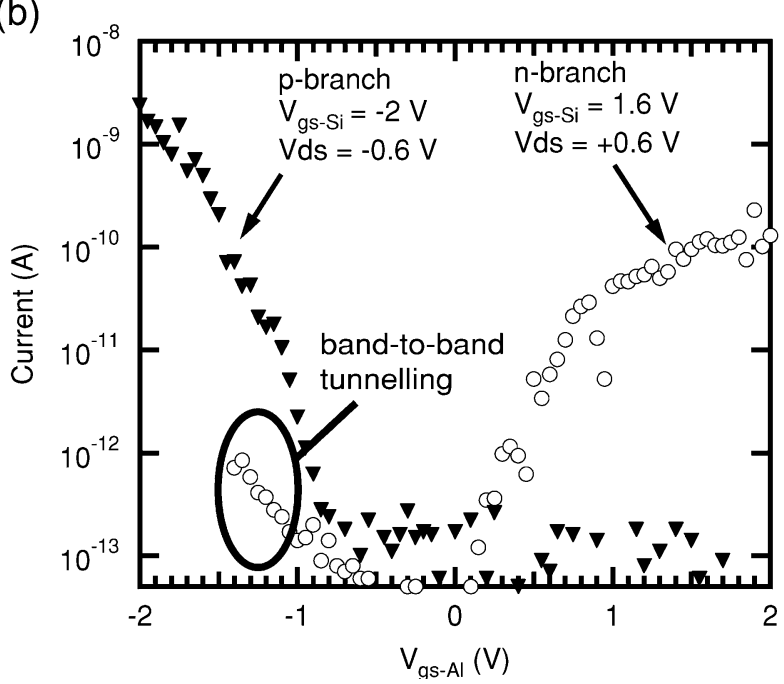

Fig. 5. (a) Subthreshold characteristics of a dual-gate CNFET at $V_{\mathrm{ds}}=-0.6 \mathrm{~V}$ when the two gate voltages are set equal $\left(V_{\mathrm{gs}-\mathrm{Si}}=\right.$ $\left.V_{\text {gs-Al }}\right)$, resembling a standard back-gated CNFET, as shown in Fig. 1(a) (b) Subthreshold characteristics $\left(I_{d}\right.$ versus $\left.V_{\mathrm{gs}-\mathrm{Al}}\right)$ of the same dual-gate CNFET measured at constant $\mathrm{Si}$ gate voltages $V_{\mathrm{gs}-\mathrm{Si}}=-2 \mathrm{~V}(\boldsymbol{\nabla})$ and $+1.6 \mathrm{~V}(\mathrm{o})$, exhibiting clear p- and n-type unipolar behaviors, respectively. Positive and negative drain voltages are used for $\mathrm{n}$ - and p-type branches, respectively, in order to illustrate the operation as regular n- and p-FET devices.

This p-FET $\left(V_{\mathrm{gs}-\mathrm{Si}}<0\right)$ and n-FET $\left(V_{\mathrm{gs}-\mathrm{Si}}>0\right)$ behavior of the dual-gate CNFET can be understood by the schematic band diagrams shown in Fig. 6(a) and (b), respectively. For a sufficiently negative (or positive) Si gate voltage, the SBs are thinned enough to allow for hole (or electron) tunneling from the metal contacts into the nanotube channel, and regions A become electrostatically doped as p-type (or n-type), resulting in a $\mathrm{p} / \mathrm{i} / \mathrm{p}($ or $\mathrm{n} / \mathrm{i} / \mathrm{n}$ ) band profile that allows only hole (or electron) transport in the nanotube channel. The dual-gate CNFET is switched ON and OFF by varying the $\mathrm{Al}$ gate voltage that alters the barrier height for carrier transport across region B. In this configuration, regions A serve as extended source and drain, and our device operates in a fashion similar to a conventional MOSFET through bulk-switching in region B. We note that for Si MOSFETs, transistors with electrically induced source/drain regions have been fabricated by using additional side gates [30], (a)

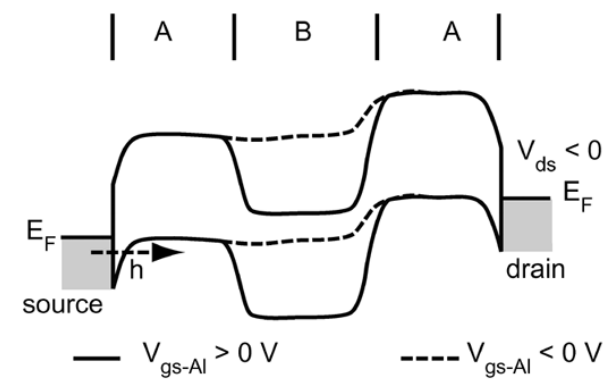

(b)

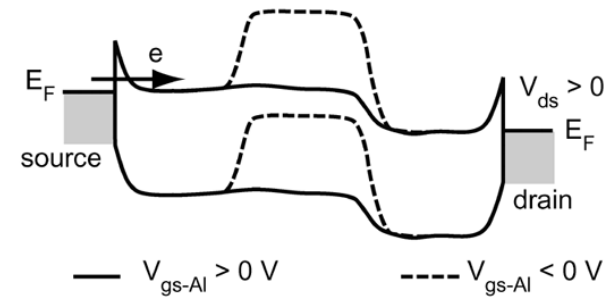

Fig. 6. Schematic band diagrams of a dual-gate CNFET for: (a) $V_{\mathrm{gs}-\mathrm{Si}}<0$ and (b) $V_{\mathrm{gs}-\mathrm{Si}}>0$. In both (a) and (b), the solid and dashed lines represent situations for $V_{\mathrm{gs}-\mathrm{Al}}>0$ and $V_{\mathrm{gs}-\mathrm{Al}}<0$, respectively.

and our dual-gate structure is reminiscent of the straddle-gate Si MOSFETs demonstrated by Tiwari et al. [31].

The results in Fig. 5(b) are in drastic contrast to the ambipolar behavior of the same device shown in Fig. 5(a), proving the successful elimination of ambipolar characteristics and the first experimental demonstration of controlling the CNFET polarity by means of electrostatic effects. It is interesting to note the n-FET branch in Fig. 5(b) exhibits a nonmonotonic behavior with the current rising for $V_{\mathrm{gs}-\mathrm{Al}} \leq-1 \mathrm{~V}$, as indicated by the circle in Fig. 5(b). This feature is due to band-to-band tunneling that occurs when the two gate voltages are different enough such that the valence band in region $\mathrm{B}$ is higher than the conduction band in regions A (or vice versa). Further discussion of this band-to-band tunneling phenomenon is reported elsewhere [35].

In our dual-gate CNFETs, the Si back gate plays an important role in determining the type of majority carrier and the device ON current. In order to study the impact of the Si gate voltage on the device performance, Fig. 7(a) shows the subthreshold characteristics of a dual-gate CNFET for different Si back-gate voltages $V_{\mathrm{gs}-\mathrm{Si}}$ with particular emphasis on the p-FET branches $\left(V_{\mathrm{gs}-\mathrm{Si}}, V_{\mathrm{ds}}<0\right)$. We first note that for $V_{\mathrm{gs}-\mathrm{Si}}>-1.0 \mathrm{~V}$, the ON current is relatively low because the current is limited by the carrier injection at the SBs rather than the barrier in region B. For $V_{\mathrm{gs}-\mathrm{Si}}=-0.5 \mathrm{~V}$, in particular, $I_{d}$ is independent of $V_{\mathrm{gs}-\mathrm{Al}}$ and the device is essentially OFF for the entire $V_{\mathrm{gs}-\mathrm{Al}}$ range, indicating the $\mathrm{SB}$ at the contact prohibits carrier emission for this $\mathrm{Si}$ gate voltage. As $V_{\mathrm{gs}-\mathrm{Si}}$ becomes more negative, the SB becomes thinner to facilitate hole injection, giving rise to a higher ON current. At sufficiently negative $V_{\mathrm{gs}-\mathrm{Si}}$ $(\leq-2 \mathrm{~V})$, the device operates as an enhancement-mode p-FET with a negative threshold voltage and an inverse subthreshold slope $S \sim 63 \mathrm{mV} / \mathrm{dec}$. We note that this $S$ value is the smallest slope reported for CNFETs to date, and is close to the theoretical minimum value of $\left(k_{B} T / q\right) \ln 10 \simeq 60 \mathrm{mV} / \mathrm{dec}$ 


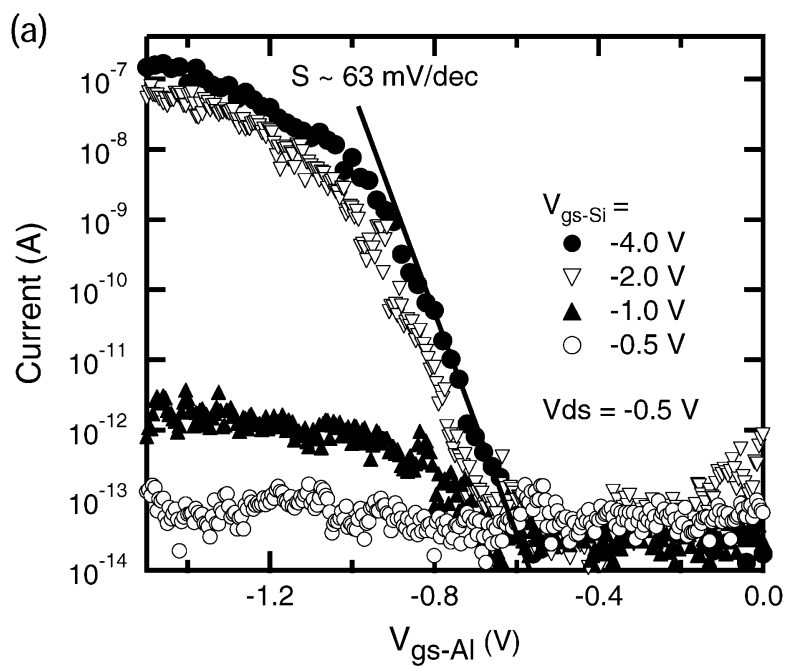

(b)

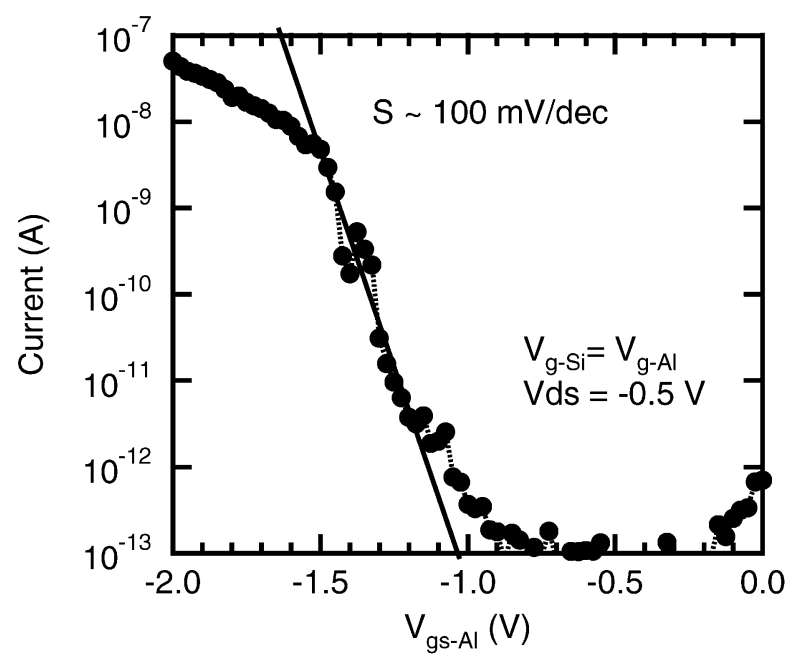

Fig. 7. (a) Measured subthreshold characteristics of a dual-gate CNFET for different Si back-gate voltages $V_{\mathrm{gs}-\mathrm{Si}}$. (b) Measure $I_{d}$ versus $V_{\mathrm{gs}-\mathrm{Al}}$ when $V_{\mathrm{gs}-\mathrm{Si}}=V_{\mathrm{gs}-\mathrm{Al}}$.

attainable for conventional MOSFETs at room temperature (where $k_{B}, T$, and $q$ are the Boltzmann constant, temperature, and electron charge, respectively). In comparison, the same device possesses $S \sim 100 \mathrm{mV} / \mathrm{dec}$ when operated as an SB CNFET with $V_{\mathrm{gs}-\mathrm{Al}}=V_{\mathrm{gs}-\mathrm{Si}}$ [see Fig. 7(b)]. The results indicate that, in addition to the elimination of ambipolar characteristics, a steeper subthreshold swing can also be obtained in our devices due to bulk switching enabled by the distinct dual-gate design. The nearly ideal $S$ value $(63 \mathrm{mV} / \mathrm{dec})$ measured for our dual-gate CNFET suggests a gate efficiency close to 1 , which is made possible by the dual back-gate design that allows exclusive Al gate control in region B. We further note that, in addition to the factors that affect the inverse subthreshold slope $S$ for conventional MOSFETs, the $S$ value of our dual-gate CNFETs also depends on tunneling properties at the SBs, as explained in the following.

In the vicinity of the SBs in region A, the carrier energy distribution $\rho(E)$ is proportional to the product of $T(E) \cdot f(E, T)$, where $T(E)$ is the tunneling probability through the SB and $f(E, T)$ is the Fermi-Dirac distribution in the metal at temperature $T$. As carriers travel away from the contact, inelastic

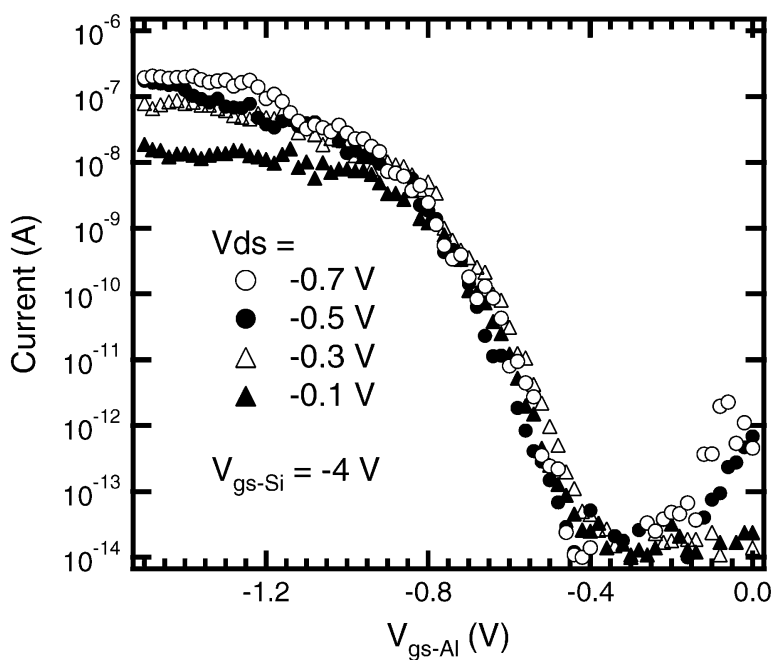

Fig. 8. Measured subthreshold characteristics of a dual-gate CNFET (same device as the one in Fig. 7) for different $V_{\mathrm{ds}}$ when the Si back-gate $V_{\mathrm{gs}-\mathrm{Si}}=$ $-4 \mathrm{~V}[28]$.

scattering processes such as electron-phonon interaction will thermalize the carrier distribution back to the equilibrium distribution characterized by the ambient temperature $T$, which is the important parameter in deriving the theoretical value of $S=\left(k_{B} T / q\right) \ln 10$. However, at low fields, the carrier mean-free-path in CNFETs can be as long as half a micrometer and the transport is essentially ballistic over the entire region A $(\sim 200 \mathrm{~nm})$. Without an effective mechanism ${ }^{4}$ to relax the nonequilibrium carrier energy distribution, the carriers arriving at the barrier in region B possess the same distribution as $\rho(E)$, which can, in principle, deviate significantly from a well-tempered Fermi-Dirac distribution if the tunneling probability $T(E)$ is highly energy-dependent. Since tunneling through an SB always favors higher energy carriers, the effective temperature $T_{\text {eff }}$ of the carrier distribution $\rho(E)$ is higher than the lattice temperature $T$ [37], causing a larger $S$ value than $\left(k_{B} T / q\right) \ln 10$. Therefore, from the aspect of device performance, an SB with $T(E) \sim \operatorname{constant}(\sim 1)$ is important not only for a high ON current, but also essential to obtain an inverse subthreshold slope close to $60 \mathrm{mV} / \mathrm{dec}$ at room temperature.

We have also studied the $V_{\mathrm{ds}}$ dependence of our device performance, as shown in Fig. 8, for the measured subthreshold characteristics of a dual-gate CNFET at different drain voltages. In Fig. 8, the Si back-gate $V_{\mathrm{gs}-\mathrm{Si}}$ is always kept at $-4 \mathrm{~V}$, and the device exhibits p-FET characteristics. Compared to the results in Fig. 1(b), the subthreshold swing of the dual-gate CNFET exhibits a much weaker dependence on the drain voltage $V_{\mathrm{ds}}$. The dual-gate CNFET possesses a very low $I_{\mathrm{off}}<100 \mathrm{fA}$, and $I_{\text {off }}$ is almost independent of $V_{\mathrm{ds}}$. This dramatic improvement of OFF-state performance for dual-gate CNFETs is not unexpected because the minority carrier injection from the drain electrode is effectively eliminated by the distinct $\mathrm{p} / \mathrm{i} / \mathrm{p}$ ( or $\mathrm{n} / \mathrm{i} / \mathrm{n}$ ) band profile in our design (see Fig. 6).

By using a novel dual-gate structure, we have successfully modified the switching mechanism of a CNFET from SB modulation to bulk-channel switching, resulting in a unipolar tran-

\footnotetext{
${ }^{4}$ We note that, for carriers acquiring energies above optical phonon energies $(\sim 180 \mathrm{meV})$, inelastic scattering can result in a carrier mean-free-path $\simeq 20-30 \mathrm{~nm}$ [36].
} 


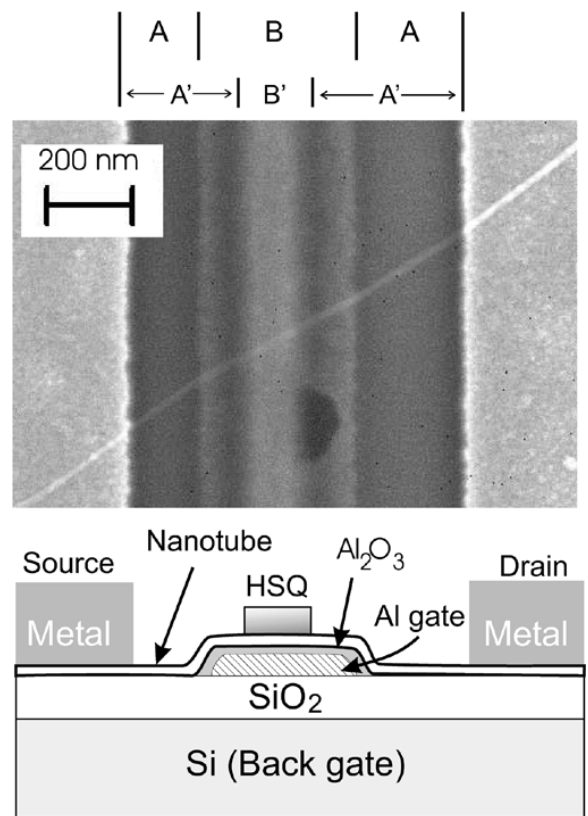

Fig. 9. (top) SEM image and (bottom) schematic cross-sectional diagram of a dual-gate CNFET with a layer of HSQ resist patterned on top of the nanotube and $\mathrm{Al}$ gate. The HSQ layer (100-nm thick), denoted as region $\mathrm{B}^{\prime}$, is used here in order to obtain a chemical doping profile along the nanotube with respect to regions $\mathrm{A}^{\prime}$ and $\mathrm{B}^{\prime}$.

sistor with tunable polarity, excellent subthreshold slopes, and a drastically improved OFF state.

\section{B. Tuning Via Chemical Doping}

In Section II-A, we have demonstrated CNFETs with improved performance due to a distinct $\mathrm{p} / \mathrm{i} / \mathrm{p}$ (or $\mathrm{n} / \mathrm{i} / \mathrm{n}$ ) potential profile along the nanotube by means of electrostatic doping in selected areas (i.e., regions A). By varying the Si back-gate voltage, the polarity of the dual-gate CNFET can be readily tuned. The role of the Si back gate, in principle, can be replaced by introducing proper chemical dopants into region $\mathrm{A}$ in order to achieve the same band bending profile. The use of chemical dopants in region A is attractive because the device can be operated with the back gate grounded $\left(V_{\mathrm{gs}-\mathrm{Si}}=0\right)$ or ultimately without the presence of any back gate, which is essential for high-frequency applications to minimize parasitic capacitances. Controlled n-type chemical doping of nanotubes has been accomplished previously by using alkali metals or air-stable polymers to create $n$-type transistors [38]-[43] and $\mathrm{p} / \mathrm{n} / \mathrm{p}$ devices [44]. However, in those devices, gating always occurs over the entire nanotube. Based on the dual-gate CNFET design, we next discuss our results on chemical doping in conjunction with electrostatic doping. In particular, n-type chemical dopants are used here to obtain an $\mathrm{n} / \mathrm{i} / \mathrm{n}$ doping profile along the nanotube.

In order to obtain a chemical doping profile along the tube, a resist layer consisting of hydrogen silsesquioxane (HSQ) is patterned on top of the $\mathrm{Al}$ gate area of a dual-gate CNFET. HSQ is a negative resist for e-beam lithography, and is compatible with our chemical doping processes for nanotubes. Fig. 9 shows the SEM image and the schematic cross section of a dual-gate CNFET patterned with a layer of HSQ resist. The HSQ layer protects the nanotube segment (region $B^{\prime}$ in Fig. 9) from being (a)

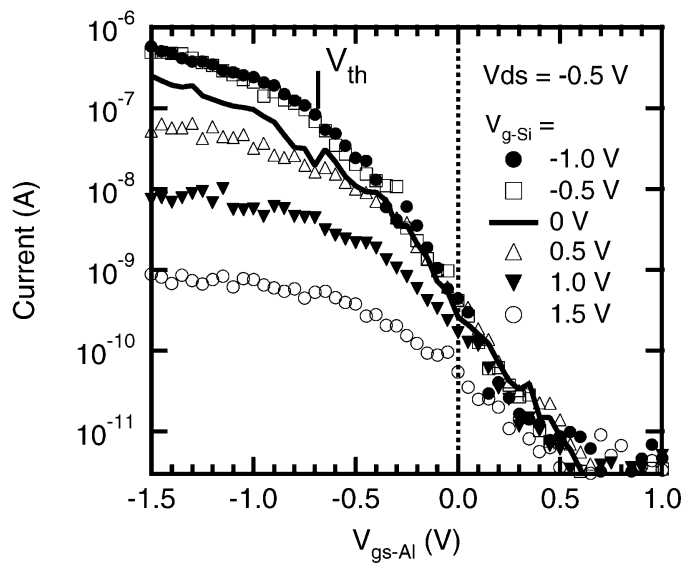

(b)

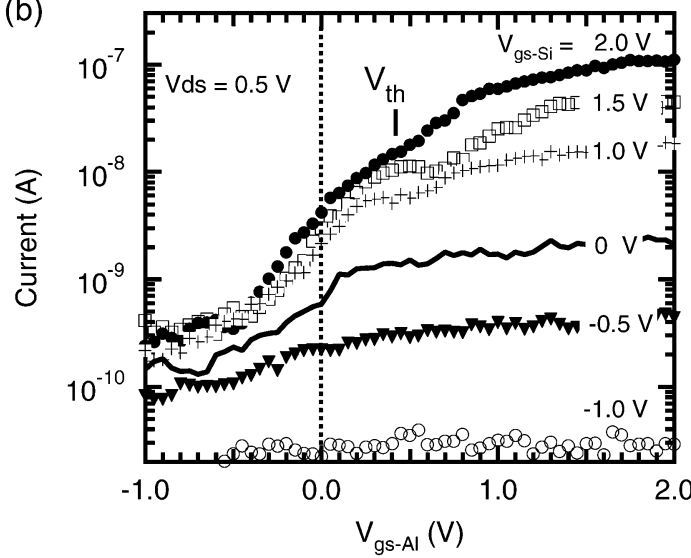

Fig. 10. Measured subthreshold swing ( $I_{d}$ versus $\left.V_{\mathrm{gs}-\mathrm{Al}}\right)$ of the CNFET (Ti/Pd contact) device, as shown in Fig. 9, for different Si back-gate voltages: (a) before and (b) after PEI doping.

influenced by chemical dopants so that a $\mathrm{p} / \mathrm{i} / \mathrm{p}$ (or $\mathrm{n} / \mathrm{i} / \mathrm{n}$ ) chemical doping profile can be obtained with respect to regions $\mathrm{A}^{\prime}$ and $\mathrm{B}^{\prime}$. In our design, the Al gate and HSQ layer permit independent electrostatic and chemical doping profiles along the tube channel.

After the HSQ patterning, Fig. 10(a) shows measured $I_{d}$ versus $V_{\mathrm{gs}-\mathrm{Al}}$ of a dual-gate CNFET (Ti/Pd contact) at different $V_{\mathrm{gs}-\mathrm{Si}}$ prior to any chemical doping treatment. ${ }^{5}$ We note that this device operates as a p-FET even for $V_{\mathrm{gs}-\mathrm{Si}} \geq 0$, suggesting that this CNFET was unintentionally p-doped during the fabrication process [11]. However, Fig. 10(a) also points out an important fact, which is that the nanotube segment in region $\mathrm{B}^{\prime}$ is not subject to this unintentional p-doping because the CNFET is still in the OFF state at $V_{\mathrm{gs}-\mathrm{Al}}=0 \mathrm{~V}$. Therefore, this CNFET possesses a $\mathrm{p} / \mathrm{i} / \mathrm{p}$ doping profile due to the unintentional p-doping effect after the HSQ patterning. The device is then dipped in an ethanol solution containing $25 \mathrm{wt} \%$ of PEI for $4 \mathrm{~h}$, followed by an ethanol rinse. Here, we use polyethylene imine (PEI), an n-type dopant for nanotubes [39], to demonstrate chemical doping effects in our devices. Fig. 10(b) shows the measured subthreshold characteristics of the same CNFET

\footnotetext{
${ }^{5}$ We note that not all dual-gate CNFETs possess an inverse subthreshold slope as steep as the one shown in Fig. 7, such as the device shown here. This could be due to the parasitic capacitance resulting from the unpassivated $\mathrm{SiO}_{2}$ and/or $\mathrm{Al}_{2} \mathrm{O}_{3}$ surfaces.
} 


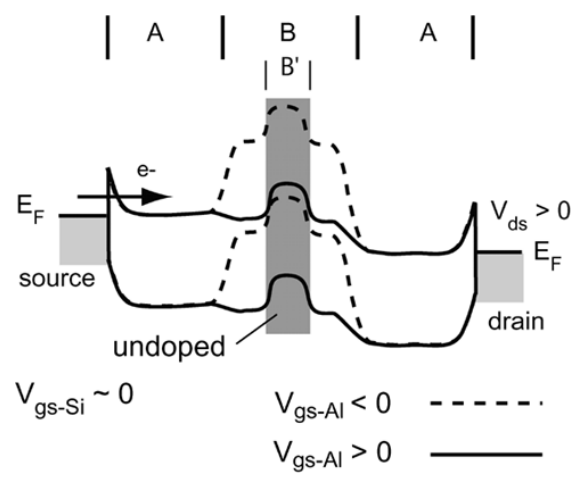

Fig. 11. Schematic band diagram of a dual-gate CNFET doped by n-type dopants in regions $\mathrm{A}$ and part of region B. The shaded area represents the undoped nanotube segment covered by the HSQ resist (region $\mathrm{B}^{\prime}$ in Fig. 9). Solid and dashed lines represent the band bending for $V_{\mathrm{gs}-\mathrm{Al}}>0$ and $V_{\text {gs-Al }}<0$, respectively.

after PEI doping, exhibiting a drastically different behavior from that of the original device shown in Fig. 10(a). The clear n-FET characteristics for $V_{\mathrm{gs}-\mathrm{Si}} \geq-0.5 \mathrm{~V}$ shown in Fig. 10(b) indicate successful p-FET to n-FET conversion by doping region $\mathrm{A}^{\prime}$ with PEI. One important feature observed in Fig. 10(a) and (b) is that both p-FET and n-FET devices before and after PEI doping operate as enhancement-mode transistors with threshold voltages $V_{\mathrm{th}}$ of approximately -0.7 and $+0.4 \mathrm{~V}$, respectively. This is due to the $\mathrm{p} / \mathrm{i} / \mathrm{p}$ and $\mathrm{n} / \mathrm{i} / \mathrm{n}$ chemical doping profile enabled by the HSQ resist layer. Without our distinct doping profile, the threshold voltage would shift with increasing doping level, resulting in a depletion-mode transistor at high doping levels [42].

The operation of the dual-gate CNFET with a chemical doping profile can be understood by the band structures depicted in Fig. 11. We note that since the area covered by HSQ resist (region $\mathrm{B}^{\prime}$ ) is narrower than the $\mathrm{Al}$ gate (region $\mathrm{B}$ ), there exists an additional band bending within region $\mathrm{B}$ where both the $\mathrm{Al}$ gate and chemical dopants are effective. This additional step in the potential profile has little effect on the transistor performance because the switching behavior is mainly determined by the total barrier height rather than the detail structure of the barrier. Band-to-band tunneling currents, on the other hand, may be substantially suppressed because of this step-like band structure in region $\mathrm{B}$.

\section{Simulation AND DisCUSSION}

We now present simulation results for our dual-gate CNFETs. In the simulation, we consider a CNFET consisting of a nanotube connected to two semi-infinite source/drain metallic contacts forming SBs. The dual-gate structure is explicitly taken into account by three gate segments between the contacts, and is characterized by lengths $L_{A}$ and $L_{B}$ for regions $\mathrm{A}$ and $\mathrm{B}$, respectively. The charge in and the current through the CNFET is calculated self-consistently using the nonequilibrium Green's function formalism together with a modified 1-D Possion equation. A quadratic dispersion relation is assumed in the conduction and valence band. Further details regarding the simulation can be found elsewhere [45].

Fig. 12 shows both measured and simulated subthreshold characteristics of a dual-gate CNFET (without chemical

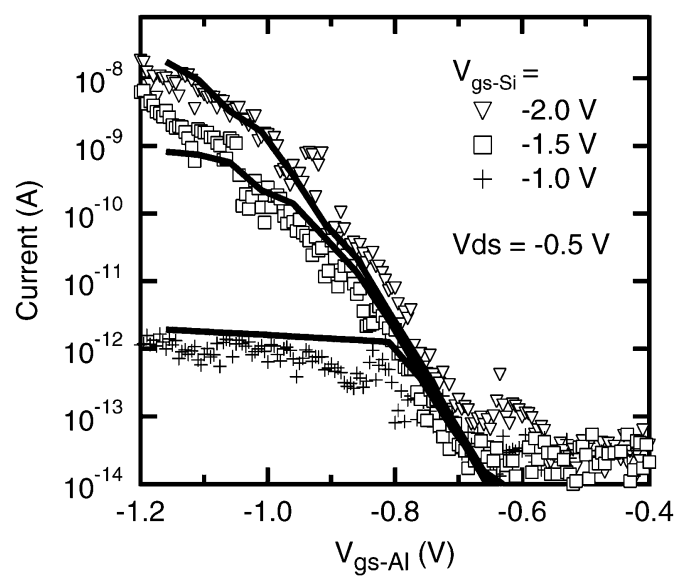

Fig. 12. Subthreshold characteristics of a dual-gate CNFET (the same device in Fig. 7) at different $V_{\mathrm{gs}-\mathrm{Si}}$. Simulated results are shown as solid curves for four Si back-gate voltages (from bottom to top): $-0.15,-0.5$, and $-1.0 \mathrm{~V}$. The simulated curves are translated by $-0.66 \mathrm{~V}$ along the $x$-axis to compare with experimental data.

doping) at different $\mathrm{Si}$ back-gate voltages. ${ }^{6}$ In the simulation, we assumed a nanotube energy gap $E_{g}$ of $1.0 \mathrm{eV}^{7}$ and an SB height $\Phi_{B}=0.3 \mathrm{eV}$ for holes. In Fig. 12, the simulated current $I_{d}$ as a function of $\mathrm{Al}$ gate voltages $V_{\mathrm{gs}-\mathrm{Al}}$ exhibits excellent agreement with measured data over a wide range of $V_{\mathrm{gs}-\mathrm{Al}}$ for various Si back-gate voltages. In particular, both experiment and simulation yield an inverse subthreshold slope $S$ close to $60 \mathrm{mV} / \mathrm{dec}$ for sufficiently negative $V_{\mathrm{gs}-\mathrm{Si}}$ values, confirming the bulk-switching phenomena in our devices. It is noteworthy that as $V_{\mathrm{gs}-\mathrm{Si}}$ varies, the simulated curves $I_{d}\left(V_{\mathrm{gs}-\mathrm{Al}}\right)$ also show good quantitative agreements with experimental data in terms of $\Delta V_{\mathrm{gs}-\mathrm{Si}}$. The ON current decreases with increasing $V_{\mathrm{gs}-\mathrm{Si}}$ because the $\mathrm{SBs}$ at the contact limit the maximum current through the device. The height of the SB is determined by the nanotube bandgap and the work function of the contact metal. It is expected that by using nanotubes of larger diameters and choosing proper metals for contact electrodes (e.g., Pd), the SBs can be effectively lowered to achieve an ON current as high as $25 \mu \mathrm{A}$ [23]. The agreement between experiment and simulation results is encouraging because it not only marks the quality of the model used here, but also provides compelling evidence for the operation concept of dual-gate CNFETs.

Since region A of our dual-gate CNFET resembles the extended source and drain in a conventional MOSFET structure, the switching speed of the dual-gate CNFET is mainly determined by the active gate in region B. For high-frequency applications, this active gate length $L_{B}$ of region B determines the transit time for an electron/hole traveling from source to drain. Therefore, although the entire length between the metal source/drain contacts is $L=2 L_{A}+L_{B}$, it is $L_{B}$ rather than $L$ that is the critical parameter in terms of lateral scaling to improve performance. In order to minimize parasitic capacitances for high-frequency applications, we have also demonstrated that the Si back gate in region A can

\footnotetext{
${ }^{6}$ In order to minimize computational time, the curves shown in Fig. 12 were, in fact, simulated with gate lengths $L_{A}$ and $L_{B}$ of $20 \mathrm{~nm}$ for both regions A and B. The gate length of $20 \mathrm{~nm}$ is found to be sufficient to describe a "long-channel" device, where the device characteristics does not depend on $L_{A}$ or $L_{B}$.

${ }^{7}$ Although the energy gap used here is larger than $E_{g} \sim 0.7 \mathrm{eV}$ typically assumed for this type of nanotube with an average diameter of $1.4 \mathrm{~nm}$, it is consistent with the value derived in [34].
} 
be replaced by using chemical dopants, although future studies are needed to quantify the correlation between the doping level and the obtained Fermi level shift.

\section{CONCLUSION}

In conclusion, we have fabricated CNFETs with a novel dualgate structure in order to achieve a distinct $\mathrm{p} / \mathrm{i} / \mathrm{p}$ or $\mathrm{n} / \mathrm{i} / \mathrm{n}$ doping profile along the nanotube. The $\mathrm{p} / \mathrm{i} / \mathrm{p}$ or $\mathrm{n} / \mathrm{i} / \mathrm{n}$ doping scheme, obtained by electrostatic and/or chemical doping effects, eliminates the ambipolar characteristics and, for the first time, creates pure $\mathrm{p}$ - or n-type enhancement-mode CNFETs with a controllable polarity for thin gate oxides. Compared to previous SB CNFETs where the SBs dominate the switching behavior, these dual-gate CNFETs are bulk-switched devices, showing the steepest subthreshold slope reported to date. The good agreement between experiment and simulation further corroborates the novel device operation concept of the dual-gate structure.

\section{ACKNOWLEDGMENT}

The authors thank Z. Chen, M. Freitag, and J. Chen for valuable discussions, and B. Ek for metal deposition and expert technical assistance, all of the IBM T. J. Watson Research Center, Yorktown Heights, NY.

\section{REFERENCES}

[1] S. Iijima and T. Ichihashi, "Single-shell carbon nanotubes of 1-nm diameter," Nature, vol. 363, pp. 603-605, 1993.

[2] D. S. Bethune, C. H. Kiang, M. S. Devries, G. Gorman, R. Savoy, J. Vazquez, and R. Beyers, "Cobalt-catalized growth of carbon nanotubes with single-atomic-layerwalls," Nature, vol. 363, pp. 605-607, 1993.

[3] M. S. Dresselhaus, G. Dresselhaus, and P. Avouris, Carbon Nanotubes: Synthesis, Structure, Properties, and Applications. Berlin, Germany: Springer-Verlag, 2001.

[4] Z. Yao, C. L. Kane, and C. Dekker, "High-field electrical transport in single-wall carbon nanotubes," Phys. Rev. Lett., vol. 84, pp. 2941-2944, 2000.

[5] M. S. Fuhrer, B. M. Kim, T. Duerkop, and T. Brintlinger, "High-mobility nanotube transistor memory," Nano Lett., vol. 2, pp. 755-759, 2002

[6] R. Martel, T. Schmidt, H. R. Shea, T. Hertel, and P. Avouris, "Singleand multiwall carbon nanotube field-effect transistors," Appl. Phys. Lett., vol. 73, pp. 2447-2449, 1998.

[7] S. T. Tans, A. R. M. Verschueren, and C. Dekker, "Room-temperature transistor based on a single carbon nanotube," Nature, vol. 393, pp. 49-52, 1998.

[8] S. J. Wind, J. Appenzeller, R. Martel, V. Derycke, and P. Avouris, "Vertical scaling of carbon nanotube field-effect transistors using top gate electrodes," Appl. Phys. Lett., vol. 80, pp. 3817-3819, 2002.

[9] A. Bachtold, P. Hadley, T. Nakanishi, and C. Dekker, "Logic circuits with carbon nanotube transistors," Science, vol. 294, pp. 1317-1320, 2001.

[10] J. Appenzeller, J. Knoch, V. Derycke, R. Martel, S. Wind, and P. Avouris, "Field-modulated carrier transport in carbon nanotube transistors," Phys. Rev. Lett., vol. 89, pp. 126801-1-126801-4, 2002.

[11] A. Javey, H. Kim, M. Brink, Q. Wang, A. Ural, J. Guo, P. McIntyre, M. Lundstrom, and H. Dai, "High- $\kappa$ dielectrics for advanced carbonnanotube transistors and logic gates," Nature Mater, vol. 1, pp. 241-246, 2002.

[12] S. Rosenblatt, Y. Yaish, J. Park, J. Gore, V. Sazonova, and P. L. McEuen, "High performance electrolyte gated carbon nanotube transistors," Nano Lett., vol. 2, pp. 869-872, 2002.

[13] G. P. Siddons, D. Merchin, J. H. Back, J. K. Jeong, and M. Shim, "Highly efficient gating and doping of carbon nanotubes with polymer electrolytes," Nano Lett., vol. 4, pp. 927-931, 2004.

[14] A. Javey, J. Guo, D. B. Farmer, Q. Wang, E. Yenilmez, R. G. Gordon, M. Lundstrom, and H. Dai, "Self-aligned ballistic molecular transistors and electrically parallel nanotube arrays," Nano Lett., vol. 4, pp. 1319-1322, 2004.
[15] R. Martel, V. Derycke, C. Lavoie, J. Appenzeller, K. K. Chan, J. Tersoff, and P. Avouris, "Ambipolar electrical transport in semiconductor single-wall carbon nanotubes," Phys. Rev. Lett., vol. 87, pp. $256805-$ 1-256 805-4, 2001.

[16] D. J. Frank, R. H. Dennard, E. Norwak, P. M. Solomon, Y. Taur, and H.-S. P. Wong, "Device scaling limits of Si MOSFETs and their application dependencies," Proc. IEEE, vol. 89, no. 3, pp. 259-288, Mar. 2001.

[17] P. L. McEuen, M. S. Fuhrer, and H. Park, "Single-walled carbon nanotube electronics," IEEE Trans. Nanotechnol., vol. 1, no. 1, pp. 78-85, Mar. 2002.

[18] P. Avouris, J. Appenzeller, R. Martel, and S. J. Wind, "Carbon nanotube electronics," Proc. IEEE, vol. 91, no. 11, pp. 1772-1784, Nov. 2003.

[19] M. Freitag, M. Radosavljevic, Y. X. Zhou, A. T. Johnson, and W. F. Smith, "Controlled creation of a carbon nanotube diode by a scanned gate," Appl. Phys. Lett., vol. 79, pp. 3326-3328, 2001.

[20] S. Heinze, J. Tersoff, R. Martel, V. Derycke, J. Appenzeller, and P. Avouris, "Carbon nanotubes as Schottky barrier transistors," Phys. Rev. Lett., vol. 89, pp. 106801-1-106801, 2002.

[21] J. Appenzeller, M. Radosavljevic, J. Knoch, and P. Avouris, "Tunneling versus thermionic emission in one-dimensional semiconductors," Phys. Rev. Lett., vol. 92, pp. 048 301-1-048 301-4, 2004.

[22] S. J. Wind, J. Appenzeller, and P. Avouris, "Lateral scaling in carbonnanotube field-effect transistors," Phys. Rev. Lett., vol. 91, pp. 0583011-058301-4, 2003.

[23] A. Javey, J. Guo, Q. Wang, M. Lundstrom, and H. Dai, "Ballistic carbon nanotube field-effect transistors," Nature, vol. 424, pp. 654-657, 2003.

[24] M. Radosavljevic, S. Heinze, J. Tersoff, and P. Avouris, "Drain voltage scaling in carbon nanotube transistors," Appl. Phys. Lett., vol. 83, pp. 2435-2437, 2003.

[25] J. Guo, S. Datta, and M. Lundstrom, "A numerical study of scaling issues for Schottky barrier carbon nanotube transistors," IEEE Trans. Electron Devices, vol. 51, no. 2, pp. 172-177, Feb. 2004.

[26] S. Heinze, M. Radosavljevic, J. Tersoff, and P. Avouris, "Unexpected scaling of the performance of carbon nanotube transistors," Phys. Rev. B, Condens. Matter, vol. 68, pp. 235 418-1-235 418-5, 2003.

[27] Y.-M. Lin, J. Appenzeller, and P. Avouris, "Ambipolar-to-unipolar conversion of carbon nanotube transistors by gate structure engineering," Nano Lett., vol. 4, pp. 947-950, 2004.

[28] — , "Novel structures enabling bulk switching in carbon nanotube FETs," in IEEE 62th Device Research Conf. Dig., 2004, pp. 133-134.

[29] A. Thess, R. Lee, P. Nikolaev, H. Dai, P. Petit, J. Robert, C. Xu, Y. H. Lee, S. G. Kim, A. G. Rinzier, D. T. Colbert, G. E. Scuseria, D. Tomanek, J. E. Fischer, and R. E. Smalley, "Crystalline ropes of metallic carbon nanotubes," Science, vol. 273, pp. 483-487, 1996.

[30] H. Kawaura, T. Sakamoto, T. Baba, Y. Ochiai, J. Fujita, S. Matsui, and J. Sone, "Transistor operation of 30-nm gate-length EJ-MOSFETs," Electron Device Lett., vol. 19, no. 3, pp. 74-76, Mar. 1998.

[31] S. Tiwari, J. J. Welser, and P. M. Solomon, "Straddle-gate transistor: Changing MOSFET channel length between off- and on-state toward achieving tunneling-defined limit of field-effect," in Int. Electron Devices Meeting Tech. Dig., 1998, pp. 737-740.

[32] A. Javey, J. Guo, D. B. Farmer, Q. Wang, D. Wang, R. G. Gordon, M. Lundstrom, and H. Dai, "Carbon nanotube field-effect transistors with integrated ohmic contacts and high- $\kappa$ gate dielectrics," Nano Lett., vol. 4, pp. 447-450, 2004.

[33] J. U. Lee, P. P. Gipp, and C. M. Heller, "Carbon nanotube p-n junction diodes," Appl. Phys. Lett., vol. 85, pp. 145-147, 2004.

[34] J. Knoch, S. Mantl, Y.-M. Lin, Z. Chen, P. Avouris, and J. Appenzeller, "An extended model for carbon nanotube field-effect transistor," in IEEE 62th Device Research Conf. Dig., 2004, pp. 135-136.

[35] J. Appenzeller, Y.-M. Lin, J. Knoch, and P. Avouris, "Band-to-band tunneling in carbon nanotube field-effect transistors," Phys. Rev. Lett., vol. 93, pp. 196 805-1-196 805-4, 2004.

[36] P. Avouris, "Carbon nanotube electronics and optoelectronics," MRS Bull., vol. 29, pp. 403-410, 2004.

[37] M. Freitag, V. Perebeinos, J. Chen, A. Stein, J. C. Tsang, J. A. Misewich, R. Martel, and P. Avouris, "Hot carrier electroluminescence from a single carbon nanotube," Nano Lett., vol. 4, pp. 1063-1066, 2004.

[38] V. Derycke, R. Martel, J. Appenzeller, and P. Avouris, "Carbon nanotube inter- and intramolecular logic gates," Nano Lett., vol. 1, pp. 453-456, 2001.

[39] M. Shim, A. Javey, N. Kam, and H. Dai, "Polymer functionalization for air-stable n-type carbon nanotube field effect transistors," J. Amer. Chem. Soc., vol. 123, pp. 11512-11513, 2001.

[40] M. Bockrath, J. Hone, A. Zettl, and P. L. McEuen, "Chemical doping of individual semiconducting carbon-nanotube ropes," Phys. Rev. B, Condens. Matter, vol. 61, pp. R10 606-R10 608, 2000. 
[41] J. Kong, C. Zhou, E. Yenilmez, and H. Dai, "Alkaline metal-doped n-type semiconducting nanotubes as quantum dots," Appl. Phys. Lett., vol. 77, pp. 3977-3979, 2000.

[42] M. Radosavljevic, J. Appenzeller, and P. Avouris, "High performance of potassium n-doped carbon nanotube field-effect transistors," Appl. Phys. Lett., vol. 84, pp. 3693-3695, 2004.

[43] J. Chen, C. Klinke, A. Afzali, and P. Avouris, "Air-stable chemical doping of carbon nanotube transistors," in 62th Device Research Conf. Dig., 2004, pp. 137-138.

[44] J. Kong, J. Cao, and H. Dai, "Chemical profiling of single nanotubes: Intramolecular p-n-p junctions and on-tube single-electron transistors," Appl. Phys. Lett., vol. 80, pp. 73-75, 2002.

[45] J. Knoch, S. Mantel, and J. Appenzeller, "Comparison of transport properties in carbon nanotube field-effect transistors with Schottky contacts and doped source/drain contacts," Solid State Electron, vol. 49, pp. 73-76, 2005.

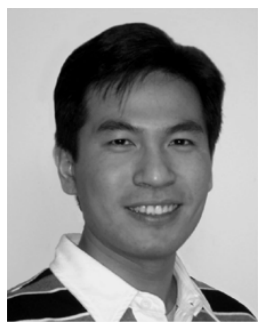

Yu-Ming Lin (M'04) received the B.S. degree in physics from National Taiwan University, Taipei, Taiwan, R.O.C., in 1996, and the M.S. and Ph.D. degrees in electrical engineering and computer science from the Massachusetts Institute of Technology (MIT), Cambridge, in 2000 and 2003, respectively. His doctoral studies focused on experimental and theoretical studies of thermoelectric properties of Bi-based nanowires fabricated using a nonlithographic self-assembly process.

In July 2003, he joined the IBM T. J. Watson Research Center, Yorktown Heights, NY, as a Post-Doctoral Fellow. His current research involves the transport study of carbon nanotube transistors.

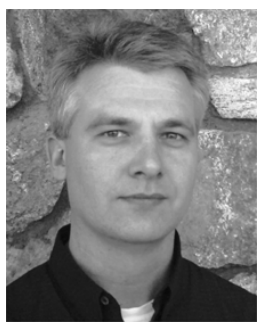

Joerg Appenzeller (SM'04) received the M.S. and $\mathrm{Ph} . \mathrm{D}$. degrees in physics from the Technical University of Aachen, Aachen, Germany, in 1991 and 1995, respectively. His doctoral dissertation concerned quantum transport phenomena in low-dimensional systems based on III/V heterostructures.

For one year, he was a Research Scientist with the Research Center Jülich, Jülich, Germany, prior to becoming an Assistant Professor with the Technical University of Aachen, in 1996. During his professorship, he explored mesoscopic electron transport in different materials including carbon nanotubes and superconductor/semiconductor-hybride devices. From 1998 to 1999, he was with the Massachusetts Institute of Technology (MIT), Cambridge, as a Visiting Scientists, during which time he explored the ultimate scaling limits of silicon MOSFET devices. Since 2001, he has been with the IBM T. J. Watson Research Center, Yorktown, NY, as a Research Staff Member, where he is mainly involved in the investigation of the potential of carbon nanotubes for future nanoelectronics.

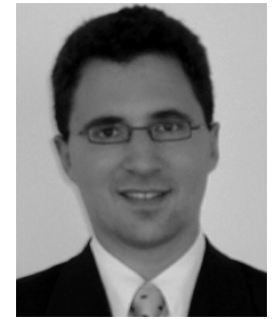

Joachim Knoch received the M.S. and Ph.D. degrees in physics from the Technical University of Aachen, Aachen, Germany, in 1998 and 2001, respectively. His doctoral studies concerned quantum transport in superconductor/semiconductor hybrids based on III-V heterostructures, as well as on the modeling and realization of ultrashort-channel MOSFETs.

From September 2001 to December 2002, he was with the Microsystems Technology Laboratory, Massachusetts Institute of Technology (MIT), Cambridge, where he was involved with InP high electron-mobility transistor (HEMT) devices. He is currently a Research Scientist with the Institute of Thin Films and Interfaces and Center of Nanoelectronic Systems, Research Center Jülich, Jülich, Germany, where he is involved in the exploration of electronic transport in alternative field-effect transistor devices such as CNFETs, ultrathin-body SB devices, and MOSFETs based upon strained silicon.

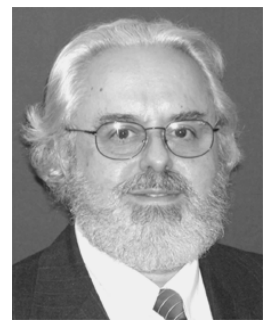

Phaedon Avouris (M'02) received the B.S. degree from the Aristotelian University, Thessaloniki, Greece, in 1968, and the Ph.D. degree in physical chemistry from Michigan State University, East Lansing, in 1994.

Following post-doctoral studies with the University of California at Los Angeles (UCLA) and AT\&T Bell Laboratories, he joined the Research Division, IBM, in 1978. He is currently an IBM Fellow and Manager of Nanometer Scale Science and Technology with the IBM T. J. Watson Research Center, Yorktown Heights, NY. He has authored or coauthored over 300 scientific papers. Over the years, his research has involved a wide variety of subjects ranging from laser studies of fast phenomena, surface physics and chemistry, scanning tunneling microscopy, and atom manipulation. His current research is focused on experimental and theoretical studies of the electrical properties and transport mechanisms of carbon nanotubes, molecules, and other nanostructures. This research includes the design, fabrication, and study of model carbon nanotube and molecular electronic devices and circuits. He is Co-Editor of the Springer-Verlag book series on "Nanoscience" and currently serves on the Advisory Editorial Boards of Nano Letters, Nanotechnology, the International Journal of Nanoscience, the Journal of Nanoengineering and Nanosystems, the Journal of Computational and Theoretical Nanoscience, Surface Review and Letters, and the Journal of Electron Spectroscopy.

Dr. Avouris is a Fellow of the American Academy of Arts and Sciences, the American Physical Society, the Institute of Physics (U.K.), the IBM Academy of Technology, the American Association for the Advancement of Science, the American Vacuum Society, and the New York Academy of Sciences. He is a member of the American Chemical Society (ACS) and the Material Research Society (MRS). He was the recipient of the Irving Langmuir Prize of the American Physical Society, the Medard W. Welch Award of the American Vacuum Society, the Feynman Prize for Molecular Nanotechnology, the Atomically Controlled Surface, Interface and Nanostructures (ACSIN) Nanoscience Prize, the IEEE Raper Award, the Distinguished Alumnus Award presented by Michigan State University, and numerous IBM Outstanding Technical Achievement awards. 